\title{
Insufficient evaluation of adverse events is not a proof of safety
}

\author{
L. G. Hemkens • U. Grouven • R. Bender • P. T. Sawicki
}

Received: 2 November 2009 / Accepted: 18 December 2009/Published online: 23 January 2010

(C) Springer-Verlag 2010

Keywords Adverse effects · Cancer · Diabetes · Glargine · Human insulin · Insulin analogue $\cdot$ Pharmacovigilance Safety

To the Editor: We read with interest the article by Home and Lagarenne [1] in which the evidence that is available to the manufacturer of insulin glargine (A21Gly, B31Arg, B32Arg human insulin) has been analysed and presented. Their conclusion is that an association of insulin glargine with an increased incidence of cancer, including breast cancer, has not been found in this analysis and that the data provide useful reassuring and contributory information regarding the safety of insulin glargine.

However, we are still not convinced about the safety of insulin glargine. The results presented are compatible with a total cancer risk increase of $36 \%$ and a breast cancer risk increase of $118 \%$ (the upper limit of the reported confidence intervals). For other cancer types such as skin cancer or gastrointestinal cancer even a fourfold increase under treatment with insulin glargine cannot be ruled out with the data presented. In principle, recent findings of a potentially increased total cancer risk of $31 \%$ during treatment with insulin glargine [2] are fully compatible with the results presented by Home and Lagarenne [1]. The results are not in disagreement with the potentially two- or threefold increased risk of breast cancer found in analyses from Sweden and Scotland [3, 4].

L. G. Hemkens $(\bowtie) \cdot$ U. Grouven $•$ R. Bender $\cdot$ P. T. Sawicki Institute for Quality and Efficiency in Health Care (IQWiG),

Dillenburger Straße 27,

51105 Cologne, Germany

e-mail: lars.hemkens@iqwig.de
Moreover, several aspects affecting the validity of the results merit closer investigation. The methods applied appear to be inadequate, as it seems that the results of all trials have simply been pooled and analysed as if the data came from one study, without the use of standard metaanalytical techniques. Assessment of trial quality and evaluation of heterogeneity are not reported and no pooled effect measure estimated by using adequate methods of meta-analysis (e.g. Peto's odds ratio) is presented. As Home and Lagarenne [1] present an analysis in which quite different trials are pooled (studies of people with type 1 and type 2 diabetes, studies of people with different age ranges, short- and long-term studies, parallel group and crossover designs, different comparators), the evaluation of heterogeneity is mandatory to produce interpretable results. Inferences about certain cancer types, especially breast cancer, are drawn using data from trials including children or with a duration of only 4 weeks. The follow-up time of the trials in the manufacturer's database is relatively short, an issue that has also been discussed concerning recently published observational studies [2-5]. In this regard the authors argue that, if the effect is based on growth promotion, cancer would appear early in the use of the agent. Following this line of argument, several trials used in their analysis would be inadequate as they overlook the relevant observation phase by including non-insulin-naive patients. Sensitivity analyses addressing all these issues are not presented.

A major issue that remains unclear is the definition of the primary outcome 'treatment-emergent neoplasm'. The authors present selected data: to allow transparent analysis and interpretation, complete data on all malignant neoplasms would be required. This is emphasised by the fact that the data used in the combined analysis 
differ substantially from those recently published by Rosenstock et al. [6]. Rosenstock et al. reported 38 events explicitly stated as malignant neoplasms during treatment with insulin glargine, and a further five events were benign or malignant (Table 2 in Rosenstock et al. [6]). Twenty-three events (malignant neoplasms) were classified as being serious in this study [6]. Home and Lagarenne [1] included only 23 serious events. However, they point out that, according to the International Conference on Harmonisation of Technical Requirements for Registration of Pharmaceuticals for Human Use (ICH) recommendation, cancer should be classified as a serious event [1]. Therefore, there needs to be clarification of why 15 out of 38 explicit malignant neoplasms in this trial were excluded or were classified as non-serious events, especially since the conclusions drawn for patients with type 2 diabetes are mainly driven by the finding of Rosenstock et al. [6]. An analysis using a meta-analytic technique (Peto approach) of the given results of type 2 diabetic patients excluding the study of Rosenstock et al. [6] shows an OR for cancer of 1.30 under treatment with insulin glargine vs comparator $(95 \%$ CI $0.68-2.49, p=0.436$; heterogeneity $I^{2}=0 \%, p=0.586$ ).

In the case of patients with type 1 diabetes it becomes apparent that the entire insulin glargine safety data from randomised controlled trials is a rather thin body of evidence (Tables 1 and 2 in the study by Home and Lagarenne [1]): three trials with a total of 285 children treated with insulin glargine, three short-term trials lasting 4-7 weeks with 420 patients treated with insulin glargine, and six trials lasting 8 months or less with about 1,000 patients treated with insulin glargine.

Unfortunately, the crucial aspect of statistical power is not addressed. The presented analysis is substantially underpowered. Even in the case of a marked increase in cancer risk with insulin glargine of $50 \%$, the probability of detecting this would be only $54 \%$ (power calculation based on a baseline cancer risk of $1 \%$ per year according to the data given in the article). This means that, with a very high probability, even a dramatic increase of cancer risk would remain undiscovered.

The data from uncontrolled studies with patients under treatment with insulin glargine are inadequately described and the adequacy of methods and reporting appears to be questionable as well. The reported US cancer incidence rate of ' 4.63 per 1,000 per year' refers to all age groups (Surveillance Epidemiology and End Results [SEER] Cancer Statistics Review [7]); in this context, the term 'age-adjusted' refers to the 2000 US Standard Population [7]. Home and Lagarenne seem to imply that the comparison between the cancer incidence in the US population and the cancer incidence in the population in the uncontrolled trials is age-adjusted [1], but this is not the case. Home and
Lagarenne compare the two incidence rates without considering an appropriate age adjustment. In these trials, which included patients with type 1 diabetes, the incidence rate refers to a probably younger population with an age structure dissimilar to that of the standard population used in SEER (in persons $<65$ years of age the corresponding cancer incidence in the USA is 2.2 per 1,000 per year [7], about one-half of incidence of 5 per 1,000 patient-years in the uncontrolled trials as reported by Home and Lagarenne [1]). In addition, the uncontrolled trials were presumably conducted worldwide, thus invalidating a direct comparison with US data for breast cancer. These indirect comparisons of the data of the uncontrolled studies do not appear to be useful and may be misleading.

Home and Lagarenne [1] found no association between insulin glargine and an increased risk of cancer, which is not surprising given the insufficient statistical power in this data set. In a situation such as this, just as the absence of evidence is not evidence of absence, absence of evidence of adverse events cannot be interpreted as proof of safety. Reporting of safety results has also been shown repeatedly to be insufficient in randomised controlled trials [8]. We think that power calculation in safety trials remains crucial before dismissing important negative outcomes.

Given the long lapse of time since approval of insulin glargine and the first reports of concerns about its safety, we expected the manufacturer's data to have been analysed and communicated more thoroughly and cautiously with analytical and methodological appropriateness, as this sensitive issue deserves. Unfortunately, in our opinion the article does not provide any valid information that would help to refute the hypothesis that insulin glargine promotes cancer growth.

Duality of interest The authors declare that there is no duality of interest associated with this manuscript.

\section{References}

1. Home PD, Lagarenne P (2009) Combined randomised controlled trial experience of malignancies in studies using insulin glargine. Diabetologia 52:2499-2506

2. Hemkens LG, Grouven U, Bender R et al (2009) Risk of malignancies in patients with diabetes treated with human insulin or insulin analogues: a cohort study. Diabetologia 52:1732-1744

3. Colhoun HM, SDRN Epidemiology Group (2009) Use of insulin glargine and cancer incidence in Scotland: a study from the Scottish Diabetes Research Network Epidemiology Group. Diabetologia 52:1755-1765. Erratum Diabetologia 52:2469

4. Jonasson JM, Ljung R, Talback M, Haglund B, Gudbjornsdottir S, Steineck G (2009) Insulin glargine use and short-term incidence of 
malignancies - a population-based follow-up study in Sweden. Diabetologia 52:1745-1754

5. Currie CJ, Poole CD, Gale EA (2009) The influence of glucoselowering therapies on cancer risk in type 2 diabetes. Diabetologia 52:1766-1777

6. Rosenstock J, Fonseca V, McGill JB et al (2009) Similar risk of malignancy with insulin glargine and neutral protamine Hagedorn (NPH) insulin in patients with type 2 diabetes: findings from a 5 year randomised, open-label study. Diabetologia 52:1971-1973

7. Horner MJ, Ries LAG, Krapcho M et al. (eds) (2009) National Cancer Institute. SEER Cancer Statistics Review 1975-2006. Available from http://seer.cancer.gov/csr/1975_2006/, accessed 19 December 2009

8. Ioannidis JP (2009) Adverse events in randomized trials: neglected, restricted, distorted, and silenced. Arch Intern Med 169:1737-1739 